\title{
Inclusión de los medios tecnológicos en el Plan de Inspección de Andalucía
}

\author{
Inclusion of ICTs in the Inspection Plan for Andalusia
}

\author{
Manuel Ángel Romero-García \\ Consejería de Educación de la Junta de Andalucía \\ maanrogar@gmail.com \\ María del Carmen Martínez-Serrano \\ Universidad de Jaén \\ mcmartin@ujaen.es
}

Recibido: 26/05/2017

Aceptado: $26 / 06 / 2017$

Publicado: 30/06/2017

\section{RESUMEN}

Este artículo muestra parte de la investigación conducente a elaborar una tesis doctoral sobre la supervisión de la acción tutorial y la orientación educativa por la Inspección de Educación de Andalucía y el uso de los medios tecnológicos previstos en el Plan de Inspección para el curso 2016/2017. La investigación se realizó con el fin de conocer en qué medida la puesta en práctica del Plan de Actuación de la Inspección Educativa está contribuyendo a la mejora de la acción tutorial y la orientación educativa y profesional, así como cuál es el grado de inclusión de los recursos TIC en las actuaciones. Para ello se diseñó un cuestionario "ad hoc" compuesto de veintisiete ítems, dividido en tres dimensiones, el cual fue cumplimentado por 98 miembros del Servicio de Inspección en las diferentes provincias que componen la comunidad autónoma. Los resultados obtenidos nos permiten conocer que la herramienta más usada es la plataforma Séneca, seguida por la plataforma Inspectio, así como un uso minoritario de la videoconferencia. A su vez, del análisis de los resultados se constata un nivel bajo de asesoramiento o supervisión sobre la integración de las TIC en relación con todos los agentes implicados en la acción tutorial (profesorado, orientadores de los IES y Servicios de Orientación Educativa).

\section{PALABRAS CLAVE}

Inspección de Educación; Plataformas Educativas; Tecnologías de la Información y la comunicación; Trabajo en red; Trabajo colaborativo.

\section{ABSTRACT}

This article is part of an ongoing research leading to a $\mathrm{PhD}$ thesis on the supervision of the educational guidance and counseling carried out by the Educational Inspection of Andalusia and whether ICTs resources are actually employed as prescribed in the Inspection Plan for the academic year 2016/2017. Research was carried out in order to assess the degree in which the actual practice of the Action Plan for the Educational Inspection is aiding to improve both educational and professional counselling and guidance; as well as attesting to the level of inclusion of ICT resources into each one of the different actions the Plan comprises. To this end, a specially made questionnaire was designed, composed of twenty-seven items split into three dimensions, being completed by ninety-eight members of the Inspection Services in each one of the provinces of the Andalusian autonomous community. The results point at the Seneca platform as the most widely used resource, followed by the Inspectio platform, as well as a minoritary use of video-conferencing. Also, results analysis makes clear the lack of advice about or supervision over ICT integration for those agents involved in counselling and guidance of students (teachers, school consellors and Educational Guidance Services).

\section{KEYWORDS}

Educational Inspection; educational platforms; ICT; networking; collaborative working. 


\section{CITA RECOMENDADA}

Romero-García, M.A. y Martínez-Serrano, M.C. (2017). Inclusión de los medios tecnológicos en el Plan de Inspección de Andalucía. RIITE. Revista Interuniversitaria de Investigación en Tecnología Educativa, 2, x-xx. Doi: http://dx.doi.org/10.6018/riite/2017/295121

\section{Principales aportaciones del artículo y futuras líneas de investigación:}

Los resultados nos conducen a nuevas vías nuevas vías de estudio en las que nos gustaría profundizar.

- Recopilar y analizar diversas experiencias sobre esta línea de investigación con la finalidad de dar visibilidad y difundir la importancia de la integración de las TIC por la Inspección Educativa.

- Investigar sobre la integración de las TIC por otros servicios de inspección a nivel nacional o internacional.

\section{INTRODUCCIÓN}

La revolución tecnológica y la rápida evolución de las Tecnologías de la Información y la Comunicación (TIC), han establecido un sistema de funcionamiento social y productivo que, sin duda, demanda cambios educativos. Lo que se ha dado en llamar la Sociedad de la Información y el Conocimiento necesita de una ciudadanía mejor preparada para acceder a la información, para comunicarse e integrar las innovaciones tecnológicas en su sistema de vida y en el tejido productivo, dotada de instrumentos para la transformación de la información en conocimiento y por tanto, competente para abordar los nuevos contextos desde la competitividad y la eficacia.

Los sistemas educativos no son ajenos a estas transformaciones. Los escenarios educativos han cambiado mediante la implementación de políticas de dotación de recursos, estrategias docentes para su aprovechamiento y la creación de nuevos entornos de aprendizaje. A su vez, la comunidad escolar está afrontando, con mayor o menor intensidad, un proceso de innovación que incluye de manera muy destacada estas tecnologías. No obstante, para el éxito de este reto es necesaria una implicación activa de todos los agentes involucrados, tanto el profesorado como los responsables de organización y gestión de los centros, pasando por los servicios de apoyo externo y la Inspección Educativa. Todo ello sin olvidar dar una especial presencia a las relaciones con las familias y al alumnado que de manera natural conviven con el mundo digital.

En una referencia expresa al caso que nos ocupa de la Inspección Educativa, Mora Baringo (2016) considera que, como cuerpo docente cuyo objetivo principal es potenciar y supervisar los procesos educativos con vista a favorecer la mejora de la calidad educativa, la Inspección no puede quedar aislada de estos procesos, no solo en sus atribuciones de supervisión, asesoramiento y control de los programas y servicios educativos, sino también en su dimensión interna.

Existen pocos trabajos de investigación que ahonden en el estudio de determinados aspectos de la labor de la Inspección Educativa. Por ello, he considerado relevante centrar la investigación en el análisis del uso de los medios que se describen en el Plan de Inspección de Andalucía, así como la valoración que los miembros del servicio de inspección hacen de la idoneidad de su actuación para propiciar el uso de la TIC por parte de los agentes educativos que son objeto de su supervisión (profesorado y agentes externos a los centros). El diseño de la investigación que se pretende tiene una finalidad descriptiva, que aspira a definir la 
valoración que hacen los miembros del Servicio de Inspección del uso de los medios tecnológicos, así como recoger aportaciones que nos permitan conocer en qué forma potencian el uso de recursos TIC en el desarrollo de estas tareas por todos los agentes implicados.

\section{EL MODELO DE INTERVENCIÓN DE LA INSPECCIÓN EDUCATIVA EN}

\section{ANDALUCÍA}

En este epígrafe vamos a describir, en primer lugar, las características específicas y diferenciadoras del modelo de intervención de la Inspección Educativa andaluza y seguidamente analizaremos algunos aspectos de las principales actuaciones del Plan de Inspección para el curso 2016/2017.

\subsection{El trabajo en red en la Inspección Educativa de Andalucía}

El modelo de intervención de la Inspección de Educación en la Comunidad Autónoma de Andalucía se desarrolla a través de unas características, unos elementos metodológicos y aspectos organizativos contenidos en lo que Luna (2014) definía como sus "señas de identidad". Este modelo se desarrolla con el objetivo de cohesionar y homologar todas las actuaciones del Plan General de Actuación, generando dinámicas de cambio en sus aspectos organizativos y en las pautas de actuación de la Inspección.

Una de las sinergias que posibilitan estas dinámicas de comunicación, colaboración e innovación o cambio es lo que en la literatura al respecto se viene denominando trabajo en red. Para ello en el Servicio de inspección de Andalucía se fomenta la integración de diversos recursos tecnológicos, siendo el más reciente y novedoso la creación de la plataforma Inspectio.

Los principios de trabajo y funcionamiento que sostienen el sistema de trabajo en red son:

- Horizontalidad

- Accesibilidad

- Funcionalidad

- Trabajo en equipo

- Descentralización

- Autonomía profesional

- Participación

- Multidireccionalidad.

Por ello, los Servicios de Inspección se están convirtiendo, cada vez más, en entornos colaborativos, donde el trabajo en equipo preside buena parte de las actuaciones. Para comprobar las evidencias de lo manifestado hasta ahora haremos un somero análisis del Plan de Inspección.

\subsection{El Plan de Inspección de Andalucía.}

En el presente apartado vamos a realizar un breve desglose de las principales actuaciones que recoge el Plan para el curso escolar 2016-17, haciendo referencia a los recursos básicos que se incluyen, los procedimientos que se aplicaran y otros aspectos que definen los cauces comunicación y organización del trabajo. 


\subsubsection{Actuación A1}

A.1.Intervención centrada en la atención personalizada del alumnado en riesgo de no obtener la titulación básica, a través de medidas preventivas y recuperadoras que eviten la ruptura de los procesos de aprendizaje o la reincorporación al sistema, mediante la supervisión de factores clave adecuados a dicha intervención.

Los recursos básicos que se implementan para el desarrollo de la actuación son:

a) La aplicación de la plataforma de gestión Séneca para el análisis de los siguientes datos en relación con los agentes implicados en el proceso educativo:

- Alumnado: gestión de la orientación, evaluación y seguimiento del absentismo.

- Centro: horarios, tasas, autoevaluación de centros.

b) Consulta de fuentes normativas, a través de la página Web ${ }^{1}$ de la Inspección Educativa de Andalucía

c) Guía para la profundización en el uso habitual de Inspectio, uso de videoconferencias, aplicaciones móviles y registro de buenas prácticas.

Entre las acciones y procedimientos a desarrollar por los implicados en la actuación se establecen:

a) Reuniones de coordinación y homologación con la Inspección General. Se plantea la posibilidad de realizar reuniones y videoconferencias, si procede, para aclarar dudas de la actuación, plantear propuestas y homologar criterios y documentación de la actuación; así como reuniones para el seguimiento de la actuación con los diferentes Servicios Provinciales.

b) Sesiones formativas a nivel general.

c) Sesiones formativas de carácter presencial sobre las nuevas leyes 39 y 40 / 2015, Leyes de transparencia y protección de datos, así como videoconferencias con las personas que se determinen y otras para la elaboración de documentos que faciliten el desarrollo de la actuación.

Un aspecto relevante dentro de la actuación es el análisis y valoración de la información obtenida del estudio del Plan de Mejora y de la Memoria de Autoevaluación de los centros.

Las fuentes que se ponen a disposición de los inspectores-as de educación y las acciones que se sugieren desde el Plan para propiciar la calidad del análisis de estos documentos son:

- Conclusiones de los Dictámenes de la Inspección Educativa de Andalucía (disponibles en Inspectio).

- Procesos de autoevaluación y plan de mejora del centro.

- Informes extraídos del Sistema de Información Séneca.

- Informes de los Consejos de Zona y sus comisiones.

- Otros documentos de los que dispongan los Servicios.

\footnotetext{
${ }^{1}$ http://www.juntadeandalucia.es/educacion/portals/web/inspeccioneducativa
} 


\subsubsection{Actuación A2}

A.2.Supervisión y asesoramiento de la organización y funcionamiento de los centros para contribuir al éxito educativo y a la prevención del abandono escolar.

Los recursos básicos que se implementan para el desarrollo de la actuación son, fundamentalmente, a través de la aplicación Séneca para realizar el análisis de datos e información básica de los centros.

Por lo que respecta a los centros concertados se analizan los datos de la jornada escolar y el horario, teniendo en cuenta la información recogida en las visitas a centros y reuniones con equipos directivos, así como los datos disponibles en Séneca, en el apartado "Centro".

Para los centros públicos se analizan los siguientes aspectos:

- Jornada: información facilitada por la dirección de los centros y obtenida en las visitas giradas.

- Horarios: información incluida en Séneca, apartado "Centro/Horarios".

- Situación de la dirección del centro: información disponible en Séneca y facilitada por la dirección de los centros.

- Supervisión de la Memoria de Autoevaluación y Plan de Mejora, a través del Módulo de Séneca sobre el Proceso de Autoevaluación y Mejora e información obtenida en las visitas a centros.

Esta actuación incluye también el análisis de los resultados de las diferentes evaluaciones. Éste se realiza utilizando como herramienta principal la aplicación Séneca.

\subsubsection{Actuación A.3}

A.3. Intervención en procesos de evaluación y autoevaluación: selección y evaluación de la función directiva; evaluación del funcionariado docente en prácticas; evaluación de servicios y Programas; participación en evaluaciones externas del sistema educativo; evaluación del alumnado y garantías procedimentales. Esta actuación prevé su desarrollo en dos fases:

En esta primera fase se prevé la integración, como mínimo, de los siguientes recursos:

- Plataformas Inspectio y Séneca.

- Videoconferencia.

En la segunda fase, "Visitas al centro, aulas o servicios", La integración de recursos es similar a la fase I, desglosamos su integración en relación con las principales acciones y procedimientos previstos en esta actuación:

1. Selección y evaluación de la función directiva.

- Utilización del módulo de Séneca en la parte correspondiente a la selección de directores.

- Para la valoración procesual de la función directiva se prevé la grabación de evidencias, a lo largo del curso, en el módulo correspondiente de Séneca.

- Grabación en Séneca del informe final de evaluación del desempeño de la dirección al finalizar mandato. 
2. Evaluación del funcionariado en prácticas.

- Propuesta y nombramientos de vocales y presidencias de Comisiones de Evaluación utilizando el módulo correspondiente de Séneca.

- Evaluación de la fase de prácticas y grabación de documentación en Séneca.

3. Supervisión y asesoramiento de procesos de autoevaluación y mejora.

- Comprobación en Séneca de la grabación de las Memorias de Autoevaluación de los Centros, instando a los centros que falten a que realicen la tarea.

- Asesoramiento a los centros sobre el desarrollo del proceso de Autoevaluación y Mejora.

\subsubsection{Actuación A.4}

A.4Supervisión y asesoramiento de la garantía del ejercicio de los derechos y deberes de la comunidad educativa, de la participación, la igualdad y la convivencia

La previsión de recursos es similar a la de otras actuaciones:

- Plataformas Inspectio y Séneca.

- Videoconferencia (para la formación de los responsables provinciales y su coordinación con la Inspección General)

\section{LA INVESTIGACIÓN}

En este apartado se describe la forma en que se plantea la investigación. Inicialmente valoraremos el contexto en el que se ha llevado a cabo la investigación y más concretamente el marco de referencia metodológico. El segundo paso será la explicación del diseño de la investigación. Seguidamente expondremos el procedimiento que se ha utilizado para la obtención de los datos. Para finalizar este apartado se describe la población y muestra con la que se trabaja, la elección de los sujetos para la conformación de la muestra y la descripción del procedimiento a seguir.

A continuación, se procede a la descripción del instrumento de recogida de información en función de las variables tenidas en cuenta para el desarrollo de la investigación, la elaboración del mismo, la formulación de las preguntas o ítems y el procedimiento de aplicación

\subsection{Contexto}

La investigación se suscribe al desarrollo del Plan de Inspección por parte de los inspectores e inspectoras que realizan su trabajo en las diferentes Delegaciones Territoriales de Educación en la comunidad autónoma de Andalucía.

\subsection{Diseño de la investigación}

La investigación parte de la reflexión sobre un problema que el investigador percibe en la eficacia del Plan de Inspección de la Consejería de Educación de la Junta de Andalucía para la implementación de las TIC en la labor de inspección del sistema. Seguidamente pasamos a delimitar el problema de la investigación. Los siguientes pasos fueron el diseño del procedimiento de la investigación, la revisión de la bibliografía al respecto, la elaboración y aplicación de un cuestionario a una muestra de inspectores e inspectoras en las diferentes provincias andaluzas. Después se procedió a verificar la validez del cuestionario. Por último, se realizó un análisis estadístico de los datos y se derivaron conclusiones e implicaciones pertinentes en función de los resultados obtenidos. 


\subsection{Procedimiento}

La investigación se ha desarrollado en dos fases: la primera ha sido una fase de revisión de la literatura referente al tema de la función de supervisión del sistema educativo que realiza la inspección y más concretamente sobre lo que respecta a acción tutorial y la orientación educativa en diferentes contextos y la segunda ha consistido en la confección del cuestionario, selección de la muestra, recogida de datos y análisis de los mismos.

La metodología empleada es fundamentalmente cuantitativa, desde una perspectiva empírica analítica. Es decir, la realidad se presenta como algo externo al investigador y la finalidad es conocerla y explicarla para poder realizar conclusiones y aportaciones que permitan mejorarla. Se parte de la teoría para luego explicar el fenómeno (Yuni y Urbano, 2003). La fundamentación teórica servirá para seleccionar y estudiar variables, además de ayudar a realizar un mejor análisis e interpretación de los datos obtenidos.

Para confeccionar el instrumento se procedió a una revisión de la literatura en bases de datos, documentos, búsquedas Web, investigaciones y publicaciones dentro de nuestro contexto y fuera de él, así como a la normativa que regula su funcionamiento y organización.

Con la información obtenida se realizó un banco de ítems, los cuales estaban relacionados con la temática objeto de la investigación y con los objetivos de la misma. De esta selección de ítems se elaboró una primera versión del cuestionario.

Seguidamente dichos instrumentos se entregaron a seis expertos para que lo depurasen y validaran. Con las aportaciones realizadas se modificó el cuestionario y se volvió a someter a una nueva revisión por otro grupo de expertos.

Una vez cumplimentados todos los cuestionarios se procedió a su análisis mediante el programa SPSS para Windows. Concretamente se estudió la fiabilidad y la validez; así como otros análisis estadísticos. Con ello se pretende demostrar que el instrumento usado para la recogida de datos cumple los requisitos de validez y fiabilidad.

\subsubsection{El instrumento de recogida de información: el cuestionario}

La finalidad de este apartado es describir el cuestionario, diseñado para recoger la opinión de los inspectores e inspectoras de educación el uso que realizan de las TIC y los recursos que la Consejería de Educación pone a su disposición para la realización de las tareas de supervisión.

Para ello, primeramente, recurrimos a la literatura a fin de informarnos sobre su elaboración. Seguidamente, pasamos a describir el cuestionario: sus dimensiones, tipo de ítems, etc. Esto nos proporcionó información relevante y asesoramiento para su diseño.

Nos decidimos por un cuestionario con una escala Likert, con los valores de 1 (nada), 2 (poco), 3 (bastante) y 4 (mucho), siendo el número par para forzar al encuestado a elegir y a reflexionar y por tanto a que no tendiera a una postura media por ser ésta la más sencilla de responder.

El cuestionario está conformado por cuatro partes diferenciadas; la primera hace referencia al objetivo que se persigue mediante la aplicación de este instrumento, así como a las instrucciones para la cumplimentación del mismo. El segundo apartado es el referido a los 
datos personales: sexo, edad formación previa al ingreso en el Servicio de Inspección. El tercer apartado hace referencia a una serie de datos profesionales: Antigüedad en el Servicio de Inspección, situación administrativa dentro del servicio, pertenencia al equipo de coordinación provincial, coordinación de algunas de las actuaciones del Plan de Inspección o de las áreas estructurales. Con el cuarto apartado se inicia el cuestionario propiamente dicho. A continuación, indicamos las dimensiones de que consta el mismo:

- Uso de las TIC por los miembros del servicio de inspección: uso de los recursos TIC en el desarrollo de las actuaciones de la Inspección Educativa.

- Fomento de la inclusión de las TIC: supervisión del adecuado uso de recursos tecnológicos y plataformas educativas (Séneca, PASEN, etc.) por parte del profesorado y responsables de la orientación educativa.

En la primera dimensión se pretende medir el uso que los miembros del Servicio de Inspección de Andalucía declaran hacer de los principales recursos que la Consejería de Educación pone su disposición para el desarrollo del Plan de Inspección y la puesta en práctica de las atribuciones de supervisión que la normativa les confiere. Se han limitado el número de recursos a aquellos, que a tenor de la literatura al respecto y la descripción de las actuaciones en las resoluciones y documentos internos (guías), se perciben o proponen como de uso más frecuente. En la segunda dimensión del cuestionario se han recogido datos que permitan valorar si los inspectores de educación en Andalucía están potenciando el uso de las TIC en el ámbito escolar.

Una vez confeccionado el cuestionario procedimos a enviar por correo o entregar en mano, según la ubicación de los Servicios Provinciales de Inspección, los cuestionarios. A cada Servicio se enviaron entre 24 y 50 cuestionarios, dependiendo del número de inspectores del mismo, así como una carta de presentación en la que se rogaba que realizaran el cuestionario al mayor número posible de miembros.

\subsection{Muestra}

La población de nuestro estudio está formada por los inspectores e inspectoras miembros del Servicio de Inspección educativa en las diferentes Delegaciones Territoriales de Educación, la cual de acuerdo con lo establecido en el Anexo del DECRETO 115/2002, de 25 de marzo, por el que se regula la organización y el funcionamiento de la Inspección Educativa, está compuesta por un total 250 miembros. No obstante, estas plantillas se han visto incrementadas por el Acuerdo de 29 de marzo de 2007 entre la Consejería y las Organizaciones Sindicales, para la mejora del funcionamiento de los centros por la aplicación de la LEA. En el apartado octavo de dicho acuerdo se recoge: "Las plantillas de Inspectores de educación se incrementarán hasta conseguir, en el periodo 2008/2012, una relación 1/15 entre el número de inspectores e inspectoras y el de centros docentes". Por lo que actualmente la distribución de los miembros en los diferentes Servicio Provinciales arroja una muestra de 276 componentes.

Tabla № 1. Población y muestra del estudio.

\begin{tabular}{|c|c|c|c|c|c|c|c|c|c|}
\hline Provincia & Almería & Cádiz & Córdoba & Granada & Huelva & Jaén & Málaga & Sevilla & Total \\
\hline $\mathbf{N}^{\circ}$ insp. & 24 & 38 & 32 & 36 & 24 & 26 & 42 & 54 & 276 \\
\hline
\end{tabular}

El tamaño de la muestra $(n=98)$ con respecto a la población total que la compone es sobradamente representativo, el $35,5 \%$ de los inspectores adscritos a los diferentes servicios 
cumplimentaron el cuestionario. En cualquier caso, el tamaño de la muestra (Fox, 1987) se determina seleccionando arbitrariamente un número o una proporción, tal como el 10 o el $20 \%$ de la población. En palabras de este mismo autor hay que resaltar la importancia que tiene la representatividad y no el tamaño de la muestra, como aspecto fundamental del muestreo. Por lo tanto, el tamaño muestra correcto es aquél que permite conseguir la precisión que el investigador desee. Incluso sería conveniente tener en cuenta los análisis estadísticos que se piensan emplear, también los procesos de recogida de datos que se utilizarán y los posibles efectos que puedan tener sobre aquellos en cuanto a la reducción de la muestra aceptante (Fox,1987, p. 397).

\section{RESULTADOS}

En el presente apartado presentamos brevemente los análisis estadísticos del cuestionario; concretamente realizamos el estudio de la fiabilidad, la validez y el análisis descriptivo básico (media, moda, desviación típica y porcentajes).

\subsection{Estudio de fiabilidad y validez}

La fiabilidad permite medir el grado de reproductibilidad de un instrumento, es decir, la capacidad de obtener los mismos resultados si éste es aplicado en situaciones distintas.

En la presente investigación para calcular la fiabilidad del cuestionario aplicamos el alfa de Cronbach. El programa estadístico que hemos empleado es el SPSS-15-0 para Windows. A continuación pasamos a describir cada uno de ellos.

En la presente investigación para calcular la validez del cuestionario utilizamos el juicio de expertos. Con este procedimiento pudimos depurar el instrumento, ya que lo corregimos, aumentamos el número de ítems de algunos apartados del cuestionario y lo redujimos en otros, a la vez que adaptamos el lenguaje a la terminología y formas de expresión más idóneas para la comprensión.

La totalidad del cuestionario ha obtenido un Alpha total de ,867 por lo que podría considerarse alto, por tanto, no ha sido necesario rectificar ningún ítem.

\subsection{Análisis descriptivo}

Iniciamos el análisis descriptivo con la valoración de los resultados de media, moda y desviación típica. Seguidamente realizamos una valoración de los resultados de los porcentajes.

\subsubsection{Resultados y discusión de la media, moda y desviación típica.}

En este apartado, pasamos a calcular la media, moda y desviación típica, de cada variable; con el fin de describir la muestra y obtener una visión en su conjunto de ésta, así como la forma de su distribución.

A continuación, exponemos los datos obtenidos de la media, moda y desviación típica (DT) de los cuestionarios realizados, así como el análisis de los resultados de los mismos. 
Tabla № 3. Media, moda y desviación típica.

\begin{tabular}{|c|c|c|c|c|c|c|c|c|}
\hline & \multicolumn{5}{|c|}{ USO DE LOS MEDIOS PREVISTOS EN EL PLAN } & \multicolumn{3}{|c|}{$\begin{array}{c}\text { ASESORAMIENTO Y SUPERVISIÓN } \\
\text { PARA LA INTEGRACIÓN DE LOS } \\
\text { MEDIOS }\end{array}$} \\
\hline & Séneca & Inspectio & Email & Teléfono & $\begin{array}{c}\text { Video- } \\
\text { conferencia }\end{array}$ & Profesorado & $\begin{array}{c}\text { Orientadores } \\
\text { IES }\end{array}$ & $\begin{array}{l}\text { Equipos de } \\
\text { Orientación }\end{array}$ \\
\hline Media & 3,46 & 2,45 & 3,41 & 3,44 & 1,71 & 2,21 & 2,18 & 2,04 \\
\hline Moda & 4,00 & 2,00 & 4,00 & 4,00 & 2,00 & 2,00 & 2,00 & 2,00 \\
\hline $\begin{array}{l}\text { Desv. } \\
\text { típ. }\end{array}$ & ,720 & ,788 & ,730 & ,719 & ,760 & ,803 & ,709 & ,772 \\
\hline
\end{tabular}

En lo que respecta a la valoración del uso de los medios recogidos en el plan de Inspección los queda claro que la plataforma Seneca es la más usada $(3,46)$. Ello puede deberse a que es la única plataforma de uso, prácticamente, obligatorio para todos los miembros del servicio, dado que es la plataforma a través de la cual se desarrolla la gestión del análisis de datos y la gestión de muchas de las tareas de supervisión (actuaciones A.2. y A.3 fundamentalmente). La otra plataforma, Inspectio, de reciente creación, utilizada para favorecer la coordinación el desarrollo del plan a través de información y difusión de las guías de las actuaciones, desarrollo de informes homologados entre otras tareas, es puntuada con un 2,45, lo que denota un uso más bien escaso. El resto de los recursos técnicos previstos en el plan son bastante usados, con excepción de la videoconferencia $(1,71)$, aunque aparece incluida en la mayoría de las actuaciones, sólo se usa como instrumento de coordinación entre los inspectores centrales y los responsables de las diferentes actuaciones o los coordinadores de las áreas estructurales.

Por lo que respecta a la desviación típica está por debajo de un sigma en todos los ítems, destacando ligeramente las respuestas relacionadas con la plataforma Inspectio y con el uso de la Videoconferencia, siendo ambos recursos los menos usados.

En relación con el apartado de asesoramiento y supervisión para la integración por parte del profesorado en general, orientadores de los institutos y Equipos de Orientación Educativa de los recursos, tanto la media como la moda nos indica que los inspectores consideran que realizan con poca frecuencia estas labores. La desviación típica esta en todos los casos por debajo de un sigma siendo más alta para el profesorado y algo más baja los orientadores.

\subsubsection{Resultados y discusión de los porcentajes}

Seguidamente se muestran los porcentajes obtenidos para cada ítem. En virtud de lo que hemos visto anteriormente, en lo que se refiere a la desviación típica, para todos los ítems esta era inferior a 1 sigma y se movía entre 0,709 , como valor más bajo, y 0,803 , como mayor índice de dispersión, podemos determinar que los porcentajes van a estar muy agrupados en las opciones altas de la tabla, para el uso de los medios, con excepción de la videoconferencia y la plataforma Inspectio, si tenemos en cuenta que la moda para la mayoría de los ítems en esta dimensión del cuestionario se situaba en 4 . Por lo que respecta a la dimensión de asesoramiento y supervisión para la integración de los medios en todos los casos la moda era de 2, por lo que podemos prever que las opciones se van a agrupar mayoritariamente en la parte baja de la tabla. 
Tabla 4. Porcentajes.

\begin{tabular}{|c|c|c|c|c|c|c|}
\hline \multirow{2}{*}{\multicolumn{3}{|c|}{ DIMENSIONES }} & \multicolumn{4}{|c|}{ PORCENTAJES } \\
\hline & & & 1 & 2 & 3 & 4 \\
\hline \multirow{5}{*}{1.} & \multirow{5}{*}{$\begin{array}{l}\text { USO DE LOS MEDIOS PREVISTOS EN EL PLAN DE } \\
\text { INSPECCIÓN }\end{array}$} & Séneca & 2 & 7,1 & 33,7 & 57,1 \\
\hline & & Inspectio & 8,2 & 49 & 32,7 & 10 \\
\hline & & Email & 1 & 11,2 & 33,7 & 54,1 \\
\hline & & Teléfono & 2 & 7,1 & 35,7 & 55,1 \\
\hline & & Videoconferencia & 44,9 & 40,8 & 12,2 & 2 \\
\hline \multirow{3}{*}{2.} & \multirow{3}{*}{$\begin{array}{l}\text { ASESORAMIENTO Y SUPERVISIÓN PARA LA } \\
\text { INTEGRACIÓN DE LOS MEDIOS }\end{array}$} & Profesorado & 18,4 & 46,9 & 29,6 & 5,1 \\
\hline & & Orientadores IES & 15,3 & 53,1 & 29,6 & 2 \\
\hline & & $\begin{array}{ll}\text { Equipos } & \text { de } \\
\text { Orientación. } & \\
\end{array}$ & 25,5 & 46,9 & 25,5 & 2 \\
\hline
\end{tabular}

Al focalizar nuestra atención en la dimensión de utilización de las plataformas y recursos técnicos observamos que Séneca es la plataforma que obtiene unos tantos porcentajes mayores tanto entre los que la usan bastante $(33,7 \%)$ como mucho $(57,1 \%)$.

Por lo que respecta a la plataforma Inspectio, casi la mitad de los encuestados (49\%) manifiestan hacer poco uso de ella, seguidos por menos más de un tercio $(32,7 \%)$ que declara hacer bastante uso de ella.

En relación con el nivel de uso de los recursos tecnológicos (teléfono, email y videoconferencia), se evidencia de los recursos que propician de una forma sencilla el contacto más inmediato son los que aparecen como más usados, con independencia de que su integración explicita en las instrucciones que desarrollan el plan no esté expresa. Así podemos ver que el teléfono es utilizado mucho $(55,1 \%)$ o bastante utilizado $(35,7 \%)$ por los miembros del servicio que cumplimentaron los cuestionarios. En esta misma línea podemos observar que el Email tiene un nivel de uso similar (mucho, 54,1\%; bastante, 33,7\%). Por el contrario, la videoconferencia aparece citada en el Plan como un recurso para actividades de coordinación de la inspección general y los responsables de las actuaciones, las áreas estructurales, las jefaturas de los servicios o los coordinadores de los equipos, por ello su uso no está establecido de forma sistemática para todos los miembros del servicio de inspección. Por lo que la gran mayoría de los encuestados declaran no hacer ningún $(44,9 \%)$ o poco $(40,8 \%)$ uso de ella.

Por lo que se refiere a la dimensión del cuestionario que valora frecuencia con la que los inspectores asesoran y supervisan la integración de los medios entre los agentes educativos que se proponen, en todos los casos se aprecia como claramente mayoritaria la opción "poco", estando entre un $53,1 \%$ para los orientadores de los IES y un $46,9 \%$ para los otros dos agentes (profesorado y equipos de orientación). No obstante, también es de destacar que alrededor de una cuarta parte si declara asesorar a los citados profesionales con bastante frecuencia $(29,6 \%$, profesorado y orientadores; $25,5 \%$, equipos de orientación).

\section{CONCLUSIONES}

Del análisis de los resultados del cuestionario, así como del estudio de la integración de los recursos que propone el Plan de Actuación podemos establecer las siguientes premisas.

Hay una relación directa entre el nivel de utilización de los recursos y la integración de estos en las diferentes actuaciones del Plan de Inspección, en el sentido que su uso sea 
imprescindible para desarrollar determinadas tareas (análisis de datos, cumplimentación de formularios de evaluación, etc).

También se evidencia un uso sistemático de aquellos recursos que son accesibles y permiten un contacto inmediato, como es el teléfono, que no aparece directamente incluido en muchas de las actuaciones Plan de Inspección, aunque pese a ello es el sistema de comunicación más utilizado.

Los recursos menos empleados son los que se utilizan como instrumentos de coordinación para situaciones estructuradas de comunicación, como es la videoconferencia.

A pesar de los esfuerzos por integrar la plataforma Inspectio, cuya finalidad principal es favorecer el trabajo colaborativo, homologado e informar y guiar el desarrollo de las diferentes actuaciones, ésta se usa poco según declaran un número muy elevado de encuestados, dado que debido a las dinámicas de comunicación en los servicios no es imprescindible acceder a la misma para desarrollar las tareas que tienen encomendadas.

Aunque la inclusión de los recursos TIC, tanto para la docencia como para la gestión de los centros y servicios educativos, es uno de los objetivos principales de la Consejería de Educación. Los inspectores e inspectoras encuestados declaran realizar muy poco asesoramiento o supervisión en esta línea en relación con todos los agentes que se sugieren en la investigación (profesorado, orientadores de los IES y Servicios de Orientación Educativa), todo ello a pesar de que en los informes homologados de algunas de las actuaciones se valora la adecuación de la integración de las TIC, en la docencia, la acción tutorial, la comunicación institucional, etc.

La implementación de las plataformas educativas y los recursos tecnológicos en los diferentes ámbitos de la labor educativa (gestión, comunicación, docencia, tutoría, etc.) viene influenciada por factores externos al individuo e impuestos por la administración en relación con las funciones que le son propias por el rol que desarrollan.

La administración educativa debe diseñar y poner en práctica dentro del plan de formación de la Inspección Educativa que potencie el uso de todos los recursos tecnológicos que pone al servicio de los inspectores-as y que, a su vez, les permita ser agentes que propicien en cambio y la innovación cuando realizan las funciones de asesoramiento y supervisión del sistema educativo.

\section{REFERENCIAS BIBLIOGRÁFICAS}

Decreto 115/2002, de 25 de marzo, por la que se regula la Organización y Funcionamiento de la Inspección Educativa en Andalucía (BOJA, núm. 37, de 30 marzo de 2002).

Fox, D .J. (1987). El proceso de investigación en educación. Pamplona: EUNSA.

Hayman, J. L. (1984). Investigación y educación. Barcelona: Paidos.

Luna Ariza, P. (2014). Las señas de identidad del modelo de intervención de la Inspección de Educación de Andalucía. Avances en Supervisión Educativa. Revista de la Asociación de Inspectores de Educación de España, 21.

Mora Baringo, V. (2008). La inspección y los retos de las nuevas tecnologías. Avances en Supervisión Educativa. Revista de la Asociación de Inspectores de Educación de España, oo 9. 
Yuni, J. Urbano, C. (2003).Técnicas para Investigar y formular proyectos de investigación. Córdoba. Argentina: Ed. Brujas.

\title{
INFORMACIÓN SOBRE LOS AUTORES
}

\author{
Manuel Ángel Romero García \\ Inspector de Educación de la Junta de Andalucía
}

Maestro de Educación Primaria con la Especialidad de Lengua Extranjera (Inglés) por la Universidad de Granada. Licenciado en Filología Inglesa por la Universidad de Granada. Director del CEIP "Parapanda", Escóznar, Granada durante los cursos 2006 al 2012. Actualmente formo parte de la plantilla del Servicio de Inspección Educativa en la Delegación Territorial de Jaén con nombramiento Provisional. Ha participado en Visitas de Estudio en Malta y Alemania dentro del Programa Erasmus. Ha publicado en revistas nacionales de reconocido prestigio.

La línea de investigación se centra en el uso de las TIC por los miembros de la comunidad educativa.

\section{María del Carmen Martínez Serrano \\ Universidad de Jaén}

Doctora en Ciencias de la Educación por la Universidad de Granada. Profesora contratada doctora en el Departamento de Pedagogía de la Universidad de Jaén. Desarrolla su docencia en los Grados de Educación así como en el Máster de Profesorado de Educación Secundaria. La línea de investigación se centra en las TIC y el Practicum. Revisora de revistas nacionales e internacionales. Ha realizado estancia de investigación y docentes Erasmus en Universidades lusas. Es coordinadora de diversos convenios Erasmus. Ha publicado en revistas nacionales de reconocido prestigio.

\section{$(\mathrm{cc})$ BY-NC}

Los textos publicados en esta revista están sujetos a una licencia de Reconocimiento 4.0 España de Creative Commons. Puede copiarlos, distribuirlos, comunicarlos públicamente y hacer obras derivadas siempre que reconozca los créditos de las obras (autoría, nombre de la revista, institución editora) de la manera especificada por los autores o por la revista. La licencia completa se puede consultar en:Licencia Creative Commons Atribución-NoComercial-Compartir por igual 4.0 Internacional. 